\title{
Repeat Stereotactic Radiosurgery for Recurred Metastatic Brain Tumors
}

In-Young Kim, M.D., ${ }^{1,2}$ Shin Jung, M.D., ${ }^{1,2}$ Tae-Young Jung, M.D., ${ }^{1,2}$ Kyung-Sub Moon, M.D., ${ }^{1,2}$ Woo-Youl Jang, M.D., Jae-Young Park, M.D., ${ }^{1}$ Tae-Wook Song, M.D., ${ }^{1}$ Sa-Hoe Lim, Ph.D. ${ }^{2}$

Department of Neurosurgery, Brain Tumor Clinic \& Gamma Knife Center, Chonnam National University Hwasun Hospital, Chonnam National University College of Medecine, Hwasun, Korea

Department of Neurosurgery, ${ }^{2}$ Chonnam National University Hospital, Chonnam National University School of Medicine, Gwangju, Korea

Objective : We investigated the outcomes of repeat stereotactic radiosurgery (SRS) for metastatic brain tumors that locally recurred despite previous SRS, focusing on the tumor control.

Methods : A total of 114 patients with 176 locally recurring metastatic brain tumors underwent repeat SRS after previous SRS. The mean age was 59.4 years (range, 33 to 85), and there were 68 male and 46 female patients. The primary cancer types were non-small cell lung cancer $(n=67)$, small cell lung cancer $(n=12)$, gastrointestinal tract cancer $(n=15)$, breast cancer $(n=10)$, and others $(n=10)$. The number of patients with a single recurring metastasis was $95(79.8 \%)$, and another 19 had multiple recurrences. At the time of the repeat SRS, the mean volume of the locally recurring tumors was $5.94 \mathrm{~mL}$ (range, 0.42 to 29.94). We prescribed a mean margin dose of $17.04 \mathrm{~Gy}$ (range, 12 to 24 ) to the isodose line at the tumor border primarily using a $50 \%$ isodose line.

Results : After the repeat SRS, we obtained clinical and magnetic resonance imaging follow-up data for 84 patients (73.7\%) with a total of 108 tumors. The tumor control rate was 53.5\% (58 of the 108), and the median and mean progression-free survival (PFS) periods were 246 and 383 days, respectively. The prognostic factors that were significantly related to better tumor control were prescription radiation dose of $16 \mathrm{~Gy}(p=0.000)$ and tumor volume less than both $4 \mathrm{~mL}(p=0.001)$ and $10 \mathrm{~mL}$ at the repeat $\mathrm{SRS}(p=0.008)$. The overall survival (OS) periods for all 114 patients after repeat SRS varied from 1 to 56 months, and median and mean OS periods were 229 and 404 days after the repeat SRS, respectively. The main cause of death was systemic problems including pulmonary dysfunction $(n=58,51 \%)$, and the identified direct or suspected brain-related death rate was around $20 \%$.

Conclusion : The tumor control following repeat SRS for locally recurring metastatic brain tumors after a previous SRS is relatively lower than that for primary SRS. However, both low tumor volume and high prescription radiation dose were significantly related to the tumor control following repeat SRS for these tumors after previous SRS, which is a general understanding of primary SRS for metastatic brain tumors.

Key Words : Metastasis · Gamma knife radiosurgery · Radiation therapy $\cdot$ Recurrence $\cdot$ Stereotactic radiosurgery.

- Received : August 25, 2017 •Revised : October 10, 2017 •Accepted : November 24, 2017

- Address for reprints : In-Young Kim, M.D.

Department of Neurosurgery, Brain Tumor Clinic \& Gamma Knife Center, Chonnam National University Hawsun Hospital, 322 Seoyang-ro, Hwasun-eup, Hwasun 58128 , Korea

Tel : +82-61-379-7666, Fax : +82-61-379-7673, E-mail : kiy87@hanmail.net

This is an Open Access article distributed under the terms of the Creative Commons Attribution Non-Commercial License (http://creativecommons.org/licenses/by-nc/4.0) which permits unrestricted non-commercial use, distribution, and reproduction in any medium, provided the original work is properly cited. 


\section{INTRODUCTION}

The local control of stereotactic radiosurgery (SRS) for metastatic brain tumors is known to be generally favorable; however, there can be local recurrences despite previous SRS, and the incidence appears to increase with increased SRS frequency and patients' longer survival from the primary cancer. Repeat SRS can be a management technique, but its value for recurrent brain metastases remains unclear ${ }^{6,9)}$. Moreover, "repeat SRS” has mainly been considered to refer to frequency and covers both locally recurring and newly developed metastases. We focused on the former and investigated the outcomes of repeat SRS for locally recurring metastatic brain tumors despite previous SRS.

\section{MATERIALS AND METHODS}

Between December 2004 and September 2016, a total of 114 patients with 176 tumors underwent repeat SRS for locally recurring metastatic brain tumors after previous SRS. The mean age was 59.4 years (range, 33 to 85), and there were 68 male and 46 female patients. The patients had undergone previous SRS, and at their previous SRS, the mean tumor volume was $3.89 \mathrm{~mL}$ (range, 0.024 to 25.47 ), and the mean prescribed marginal radiation dose was $18.9 \mathrm{~Gy}$ (range, 12 to 24). The mean duration from the first to the second SRS was 9.1 months (range, 2.5 to 58.3). The cancer types were non-small cell lung cancer (NSCLC; $n=67)$, small cell lung cancer $(n=12)$, gastrointestinal tract cancer $(n=15)$, breast cancer $(n=10)$, and others $(n=10)$. The number of patients with single recurring metastases was 95 (79.8\%), and the other 19 had multiple recurrences. We excluded patients who had undergone previous whole brain radiation therapy (WBRT) or brain surgery, and we included patients who had undergone SRS as the primary treatment for their brain metastases.

The diagnosis for local recurrence after the SRS was mainly based on tumors that grew in volume by more than $120 \%$, and for some patients, we particularly used special MR studies including perfusion magnetic resonance imaging (MRI), MR spectroscopy, and methionine positron emission tomography (PET) to distinguish these tumor recurrences from radiation necrosis following SRS.

\section{Repeat SRS}

All patients had recurring metastatic tumors that showed contrast enhancement. Some patients received previously checked methionine PET for treatment with image fusion. Highly conformal and selective dose plans (Leksell Gamma $\operatorname{Plan}^{\circledR}$, Stockholm, Sweden) were created.

A total of 176 tumors had been treated with repeat SRS; in some patients, newly developed metastases were also treated simultaneously, but we did not include these data in this investigation.

At the time of the repeat SRS, the mean volume of the locally recurring tumors was $5.94 \mathrm{~mL}$ (range, 0.42 to 29.9). We prescribed a mean margin dose of $17.04 \mathrm{~Gy}$ (range, 12 to 24) to the isodose line at the tumor borders, and we mainly used a 50\% isodose line. Considering the possible radiation hazard due to the repeat SRS to the same brain area, 1 or 2 Gy prescription dose reduction was considered. After SRS, all patients underwent serial contrast-enhanced MRI scans that we requested every 3 months.

\section{Statistics}

SPSS version 20.0 (IBM, Chicago, IL, USA) was used to demonstrate all statistical analyses. Log-rank test was used for univariate analysis and Cox-regression analysis for multivariate analysis. The level of significance was set at $p<0.05$.

\section{RESULTS}

\section{Tumor control}

After the repeat SRS, we obtained clinical and MRI followup data for 84 patients $(73.7 \%)$ with a total of 108 tumors that had undergone repeat SRS. We considered local control failure to be when a tumor showed volumetric progression more than $120 \%$ in the latest MR images. The tumor control rate was 53.5\% (58 out of 108), and the median and mean PFS periods were 246 and 383 days, respectively, 1 year after the last patients had been registered. Among the 50 tumors that had progressed, two had been diagnosed as radiation necrosis using methionine PET.

We attempted to identify prognostic factors related to tumor control with meaningful PFS after repeat SRS using the Kaplan-Meier method (Table 1), and high prescription radiation doses during SRS were related, specifically. The tumors using 
Table 1. Univariate analysis of prognostic factors related to tumor control after repeat SRS

\begin{tabular}{|c|c|c|}
\hline Variable & Median PFS (days) & $p$-value \\
\hline Prescription dose ( $\geq 16$ vs. <16 Gy) & $735 \pm 155$ vs. $243 \pm 59$ & $0.000^{*}$ \\
\hline Prescription dose ( $\geq 20$ vs. $<20$ Gy) & $814 \pm 104$ vs. $675 \pm 91$ & 0.107 \\
\hline Tumor volume ( $\leq 4$ vs. $>4$ mL) & $998 \pm 106$ vs. $395 \pm 50$ & $0.001^{*}$ \\
\hline Tumor volume ( $\leq 10$ vs. $>10 \mathrm{~mL}$ ) & $525 \pm 168$ vs. $287 \pm 88$ & $0.008^{*}$ \\
\hline Interval from the 1st SRS ( $\geq 6$ vs. $<6$ months) & $450 \pm 51$ vs. $645 \pm 229$ & 0.805 \\
\hline Tumor location (supratentorium vs. cerebellum) & $633 \pm 63^{\dagger}$ vs. $955 \pm 192^{\dagger}$ & 0.666 \\
\hline Primary cancers (lung vs. others) & $428 \pm 46$ vs. $645 \pm 185$ & 0.773 \\
\hline Primary cancers (NSCLC vs. others) & $435 \pm 99$ vs. $525 \pm 131$ & 0.701 \\
\hline
\end{tabular}

*Statistically significant value. ${ }^{\dagger}$ Mean value. SRS : stereotactic radiosurgery, PFS : progression-free survival, NSCLC : non-small cell lung cancer

prescription doses of $\geq 16$ Gy showed longer median PFS periods ( $735 \pm 155$ days) than tumors using prescription dose of $<16$ Gy (243 \pm 59 days), and the result was clearly statistically significant $(p=0.000)$. The results at the cut-off value of 20 Gy were not statistically significant $(p=0.107)$, but the tumor control with prescription doses of $\geq 20$ Gy showed longer mean PFS periods ( $814 \pm 104$ days) than tumors which were treated with prescription doses $<20$ Gy (675 \pm 91 days). Tumor volumes $\leq 4 \mathrm{~mL}$ showed better results (mean PFS, $998 \pm 106$ days) than those for tumors $>4 \mathrm{~mL}$ (mean PFS, $395 \pm 50$ days), which was also statistically significant $(p=0.001)$. With tumor volumes of $10 \mathrm{~mL}$, the difference in tumor control was also significant $(p=0.008)$. The mean and median PFS periods for the tumors $\leq 10 \mathrm{~mL}$ were $834 \pm 87$ and $525 \pm 168$ days, and the rates for tumors $>10 \mathrm{~mL}$ was $309 \pm 70$ and $287 \pm 88$ days, both respectively.

The interval from the first SRS to the local recurrence was not a significant prognostic factor for tumor control after repeat SRS $(p=0.805)$ : tumor control was similar in the groups whose tumors recurred more than 6 months later and whose tumors recurred within no more than 6 months, and the median PFS periods were $450 \pm 51$ and $645 \pm 229$ days, respectively. Tumor location was not a significant prognostic factor $(p=0.666$ ), but the mean PFS period for supratentorial tumors (633 \pm 63 days) was shorter than that for infratentorial tumors ( $955 \pm 192$ days). Lung cancer as the primary cancer had shorter PFS periods (mean, $634 \pm 66$ days; median, $428 \pm 46$ days) than did other primary cancers (mean, $849 \pm 162$ days; median, $645 \pm 185$ days), but the differences were not statistically significant $(p=0.773$ ). NSCLC as the primary cancer had shorter PFS periods (mean, $660 \pm 72$ days; median, $435 \pm 99$ days) than the others (mean, $771 \pm 134$ days; median, $525 \pm 131$ days), but those differences were also not statistically significant $(p=0.701)$.
In the multivariate analysis, both prescription dose and tumor volume were also significantly related to tumor control, and the $p$-value were 0.006 and 0.028 , respectively. Similar to univariate analysis, any other factors related to tumor control investigated in this study were not statistically significant, either (Table 2).

\section{Managing local recurrence and new metastasis after repeat SRS}

After the repeat SRS, 50 tumors (42.3\%) in 40 patients showed local progression. Among these, two tumors in two patients were diagnosed as radiation necrosis, 33 tumors in 27 patients were tumor recurrences. However, for the other progressed 15 tumors in 11 patents, we could not make a differential diagnosis.

To manage these patients, 3rd SRS ( $\mathrm{n}=18)$, WBRT $(\mathrm{n}=4)$, and surgery $(n=7)$ were performed. However, for 11 patients including the two radiation necrosis patients, simple palliative management was indicated considering the patients' poor performance status owing to aggravated systemic cancer or radiation necrosis.

Newly developed metastases after the repeat SRS were detected in 23 patients. A 3rd SRS was performed in 10 patients, and six patients underwent WBRT. For seven other patients, simple palliation was provided considering their poor general condition.

\section{Survival after repeat SRS}

The overall survival periods for all 114 patients after repeat SRS varied from 1 to 56 months, and the median and mean OS periods were 229 and 404 days after the repeat surgeries, respectively. Fifteen patients (13.2\%) were still alive 1 year after 
Table 2. Multivariate analysis of prognostic factors related with tumor control and OS after repeat SRS

\begin{tabular}{|c|c|c|c|}
\hline Variable & Hazzard ratio & $p$-value & $95 \% \mathrm{Cl}$ \\
\hline \multicolumn{4}{|l|}{ Tumor control-related factors } \\
\hline Prescription dose ( $\geq 16$ Gy) & 0.393 & $0.006^{*}$ & $0.203-0.761$ \\
\hline Tumor volume ( $\leq 4 \mathrm{~mL})$ & 0.511 & $0.028^{*}$ & $0.280-0.931$ \\
\hline Interval from the 1st SRS ( $\geq 6$ months) & 1.053 & 0.866 & $0.579-1.915$ \\
\hline Tumor location (supratentorium) & 1.158 & 0.690 & $0.563-2.382$ \\
\hline Primary cancer (lung) & 1.179 & 0.624 & $0.611-2.276$ \\
\hline \multicolumn{4}{|l|}{ OS-related factors } \\
\hline Age ( $\leq 65$ years) & 1.246 & 0.416 & $0.733-2.118$ \\
\hline Primary cancer (lung) & 1.739 & 0.090 & $0.917-3.298$ \\
\hline Number of Mets that underwent repeat SRS (single) & 1.237 & 0.568 & $0.596-2.569$ \\
\hline Interval from the 1st SRS ( $\geq 6$ months) & 1.035 & 0.893 & $0.626-1.711$ \\
\hline Patients with controlled tumors after repeat SRS ${ }^{\dagger}$ & 1.303 & 0.307 & $0.784-2.164$ \\
\hline Patients with new metastasis after repeat SRS ${ }^{\dagger}$ & 1.105 & 0.736 & $0.620-1.968$ \\
\hline
\end{tabular}

References : <16 Gy, tumor volume $>4 \mathrm{~mL}$, interval from the 1st SRS $<6$ months, tumor location cerebellum, primary cancer others, age $>65$, number of metastasis that underwent repeat SRS multiple, patients with progressed tumors after repeat SRS, patients with new metastasis after repeat SRS. *Statistically significant value. ${ }^{\dagger}$ Statistics in 84 patients with follow-up magnetic resonance imaging after repeat SRS. OS : overall survival, SRS : stereotactic radiosurgery, $\mathrm{Cl}$ : confidence interval, Mets : metastatic brain tumors

Table 3. Univariate analysis of prognostic factors related to OS after repeat SRS

\begin{tabular}{llc}
\hline Variable & Median PFS (days) & $p$-value \\
\hline Age ( $\leq 65$ vs. $>65$ years) & $270 \pm 60$ vs. $248 \pm 50$ & 0.861 \\
\hline Primary cancer (lung vs. others) & $248 \pm 44$ vs. $230 \pm 98$ & 0.955 \\
\hline Primary cancer (NSCLC vs. others) & $258 \pm 40$ vs. $230 \pm 57$ & 0.911 \\
Number of Mets that underwent repeat SRS (single vs. multiple) & $248 \pm 43$ vs. $230 \pm 84$ & 0.508 \\
\hline Interval from the 1st SRS ( $\geq 6$ vs. $<6$ months) & $338 \pm 37$ vs. $248 \pm 21$ & 0.810 \\
Patients with controlled vs. progressed tumors after repeat SRS* & $300 \pm 56$ vs. $341 \pm 61$ & 0.541 \\
\hline Patients with new Mets after repeat SRS vs. Others* & $270 \pm 80$ vs. $321 \pm 43$ & 0.801 \\
\hline
\end{tabular}

*Statistics in 84 patients with follow-up magnetic resonance imaging after repeat SRS. OS : overall survival, SRS : stereotactic radiosurgery, PFS : progression-free survival, NSCLC : non-small cell lung cancer, Mets : metastatic brain tumors

the patient's last repeat SRS. Using the Kaplan-Meier method, we attempted to identify prognostic factors related to the patients' OS after the repeat procedures. There was no significant difference in the median survival period after the repeat SRS between younger (age $\leq 65)$ and older (age $>65)$ patients $(270 \pm$ 60 vs. $248 \pm 50$ days; $p=0.861$. By primary cancer type, neither lung cancer ( $248 \pm 44$ vs. $230 \pm 98$ days; $p=0.955$ ) nor NSCLC ( $258 \pm 40$ vs. $230 \pm 57$ days; $p=0.911$ ) showed statistically significant differences in survival after the repeat SRS, and we found no significance $(p=0.508)$ between the patients who underwent SRS for single ( $248 \pm 43$ days) versus multiple ( $230 \pm 84$ days) local recurrent metastases. The interval from the 1st SRS to the local recurrence (longer than 6 months vs. no longer than 6 months) was not a significant predictor of OS after repeat SRS ( $p=0.810$ ). The survival periods were similar between the longduration (longer than 6 months) and short-duration (no longer than 6 months) groups; the median survival rates were $338 \pm 37$ and $248 \pm 21$ days, respectively.

We also attempted to identify radiological prognostic factors related to OS in 84 patients with follow-up MRI after repeat SRS. In this group, the mean and median survival periods after the repeat SRS were 271 and 443 days, respectively. Using the Kaplan-Meier method, we found that the patients with controlled recurred tumors after the repeat SRS had a similar me- 
dian survival period ( $300 \pm 56$ days) to that for the patients with progressing recurrent tumors ( $341 \pm 61$ days), although the difference was not statistically significant $(p=0.541)$. The occurrence of new metastasis after the repeat SRS was not related to OS after the repeat SRS ( $p=0.811)$. However, the median survival in the patients without newly developed metastasis (321 \pm 43 days) was longer than that in the patients with new metastasis after the repeat SRS (270 \pm 80 days) (Table 3).

Similar to univariate analysis, any factors related to OS investigated in this study were not statistically significant, either (Table 2).

\section{Causes of death}

We investigated the causes of death in 114 patients who died, and the main cause was systemic problems including pulmonary dysfunction $(\mathrm{n}=58,51 \%)$. Three patients died of increased intracranial pressure due to uncontrolled brain metastasis. Nineteen patients (17\%) showed suspected brain-related death, which means that the brain metastases might have been symptomatic and related to the patients' poor status at the time of death according to the brain images and medical records at the time. Unfortunately, we could not identify the cause of death in 34 patients (30\%).

\section{DISCUSSION}

Although the favorable local control rate of SRS for brain metastasis has been reported as 1 year $>90 \%$, improvements in patients' survival made local failure after SRS an important clinical issue ${ }^{2,4,5,7,10,12,13)}$. Moreover, the use of SRS increased because of more indications such as increased maximum number of metastases for SRS and postoperative applications called tumor-bed SRS. For brain metastasis that recurred after a previous SRS, direct resection, radiation therapy, repeat SRS, and simple palliation can be applied ${ }^{10)}$, and among these, repeat SRS can be used for both locally recurring and newly developed brain metastases. The feasibility of repeat treatment could be one of the powerful merits of SRS compared with other treatment modalities for brain metastasis. Therefore, repeat SRS may be reasonable for patients who have both locally recurring and new metastases because it can manage both types of lesions simultaneously with minimized radiation exposure to the normal brain $^{10)}$. In our gamma knife center, 3351 SRS procedures were performed over the last 13 years, and among these, 1376 patients underwent SRS for brain metastasis. Among the brain metastasis patients, 247 underwent 2nd SRS, 36 underwent their 3rd radiosurgeries, six their 4 th, and even a 5 th for one patient. One of the advantages of SRS is that the procedure can be repeated for both recurrent and new metastases with more meaningful feasibility, effectiveness, and safety than other treatment modalities $^{6}$. However, only a few papers have reported on repeat SRS for recurrent metastasis following earlier SRS $S^{1,3,6,8-11,14)}$, and moreover, they focused on the results of the repeat SRS itself and the targeted brain metastases included both locally recurring and newly developed tumors after the first SRS, which are clearly pathophysiologically different.

With a different perspective, repeat SRS for locally recurring tumors after previous SRS might be regarded as a controversy based on the theories that doctors are using the same treatment that was previously ineffective at controlling the disease; therefore, further study on the subject is warranted. In the present study, we focused on the outcomes of repeat SRS for locally recurring metastases after previous SRS, primarily tumor control and the related prognostic factors. Additionally, we introduced brain management for both 2nd local recurrences and new metastases after the repeat SRS. Lastly, we investigated the patients' survival after the repeat SRS and related prognostic factors.

The tumor control rate in this study was only $53.5 \%$, which is relatively low, and the median PFS period was 8.2 months, both lower than the general results for primary SRS for metastatic brain tumors. However, we could expect these results because all the treated tumors had recurred after previous SRS, and they could be considered tumors that had survived the previous irradiation. Repeat SRS for these tumors re-irradiates the tumors that recurred despite the previous primary irradiation and could be expected to show poorer results than those for SRS as the primary treatment. However, in this study, repeat SRS was a kind of salvage treatment, and the median PFS of 8.2 months was not a short duration considering the OS period of 9.1 months from the repeat SRS in this patient group.

Recently, McKay et al. ${ }^{10)}$ reported the results of repeat SRS for prior local failure of SRS in 32 and 46 patients and tumors, respectively. The authors observed local recurrence in nine tumors and radiation necrosis in 11 tumors after the repeat SRS, and the 1-year control rate was 79\%. That study's authors suggested that repeat SRS for locally recurring metastatic brain 
tumors after previous SRS could be an effective salvage treatment for select patients, but there might be a higher risk of radiation necrosis. The highlight of their paper was the prediction of radiation necrosis after repeat SRS, and that occurred in tumors that had received 40 Gy or more, which we did not investigate.

However, in the present study, we considered more patients and tumors, specifically, 84 patients with 108 tumors. Moreover, we studied only patients who had received SRS; they never underwent standard surgery or WBRT for their metastatic brain tumors before the repeat SRS. Our tumor control rate of $53.5 \%$ (58 out of 108) with a median PFS period of 246 days was relatively low, but it could be expected. We did not separate tumor recurrence from radiation necrosis after the repeat SRS, and we determined tumor control failure to be when the tumor volume increased $120 \%$ compared with the volume at the repeat SRS during the regular MRI follow-up. Therefore, in addition to the two radiation necrosis patients, there might have been some radiation necrosis among the patients who received simple palliation.

In the study by McKay et al. ${ }^{10)}$, nine tumors were recurring and 14 were symptomatic of radiation necrosis. Therefore, half of the tumors (23 of 46) could have been local failure to control tumor volume ${ }^{10)}$. Their tumor control result was similar to ours, $46.5 \%$ (50 of 108 tumors).

Based on our results and the report by McKay et al. ${ }^{10)}$, tumor control following repeat SRS for locally recurring metastatic brain tumors after previous SRS is low, less than 50\%, and additional important findings were prognostic factors related to the tumor control after repeat SRS. In fact, we found that tumor volume and prescription radiation dose were significant prognostic factors in tumor control following repeat SRS for local metastatic tumors that recurred despite a previous SRS. Most physicians would likely expect our results, but we specifically presented statistical findings for a large number of patients and tumors.

The PFS period in this study could be considered favorable considering the OS after the repeat SRS among our patients. The expected median PFS period was 383 days, and the expected median OS periods for both all patients and radiologically followed patients were 404 and 443 days, respectively. The identified causes of death in more than half of the patients were systemic problems, and direct brain-related death was low. Therefore, repeat SRS for recurring metastatic tumors after a previous SRS could be an option for palliative management in these patients. However, we could not find significant prognostic factors related to patients' OS.

The present study does have some limitations, the first being the lack of patients' functional outcomes. Of course, the Karnofsky performance scores (KPS) at the repeat SRS and at the last follow-up imaging were 70 or higher; however, we could not investigate how long these KPS scores after the repeat SRS because of the lack of information in the medical records; future studies should include the functional outcomes after the repeat SRS.

Second, we did not radiologically distinguish 2 nd local recurrences from radiation necrosis unless patients needed $3 \mathrm{rd}$ interventions because of their poor general conditions or primary cancer status. Therefore, we believe that some of the tumors that progressed after the repeat SRS could have been radiation necrosis considering the reported relatively high incidence in a similar patient group ${ }^{10)}$.

Third, we did not consider the additional effect of systemic treatment. However, we think that medical oncologist would not change the chemotherapy regimen or start a new chemotherapy treatment after identifying locally recurred brain metastasis, even though they change or start again a systemic treatment when first detection of brain metastasis or newly developed brain metastasis. And, the locally recurred brain metastasis in the patients despite maintenance of keeping chemotherapy could indicate no effect of those chemotherapeutic agents on preventing the local recurrence of brain metastasis.

Lastly, the patients in this study were patients whose general condition was relatively good and primary cancer status was sufficiently stable for them to undergo repeat SRS. That is, there may have been bias in the results for the patients' survival in this study.

\section{CONCLUSION}

Although the tumor control of repeat SRS for locally recurring metastatic brain tumors after a previous SRS was lower than that following primary SRS, the control rate and PFS were meaningful considering the patients' OS after the repeat SRS.

One of the important findings in this investigation was that both tumor volume and prescription radiation dose were significant prognostic factors for tumor control following repeat 
SRS for locally recurring metastatic brain tumors after previous SRS, which is already established for primary SRS for metastatic brain tumors. Therefore, regular MRI follow-up after the 1st SRS is important for early detection of smaller local recurrences and for consecutive SRS with high radiation doses.

\section{CONFLICTS OF INTEREST}

No potential conflict of interest relevant to this article was reported.

\section{INFORMED CONSENT}

Informed consent was obtained from all individual participants included in this study.

\section{References}

1. Ammirati M, Cobbs CS, Linskey ME, Paleologos NA, Ryken TC, Burri SH, et al. : The role of retreatment in the management of recurrent/progressive brain metastases: a systematic review and evidence-based clinical practice guideline. J Neurooncol 96 : 85-96, 2010

2. Baschnagel AM, Meyer KD, Chen PY, Krauss DJ, Olson RE, Pieper DR, et al. : Tumor volume as a predictor of survival and local control in patients with brain metastases treated with gamma knife surgery. J Neurosurg 119 : 1139-1144, 2013

3. Chen JC, Petrovich Z, Giannotta SL, Yu C, Apuzzo ML : Radiosurgical salvage therapy for patients presenting with recurrence of metastatic disease to the brain. Neurosurg 46 : 860-866; discussion 866-867, 2000

4. Cho KR, Lee MH, Kong DS, Seol HJ, Nam DH, Sun JM, et al. : Outcome of gamma knife radiosurgery for metastatic brain tumors derived from non-small cell lung cancer. J Neurooncol 125 : 331-338, 2015
5. Jensen CA, Chan MD, McCoy TP, Bourland JD, deGuzman AF, Ellis TL, et al. : Cavity-directed radiosurgery as adjuvant therapy after resection of a brain metastasis. J Neurosurg 114 : 1585-1591, 2011

6. Kwon KY, Kong DS, Lee JI, Nam DH, Park K, Kim JH : Outcome of repeated radiosurgery for recurrent metastatic brain tumors. Clin Neurol Neurosurg 109 : 132-137, 2007

7. Lucas JT Jr, Colmer HG 4th, White L, Fitzgerald N, Isom S, Bourland JD, et al. : Competing risk analysis of neurologic versus nonneurologic death in patients undergoing radiosurgical salvage after whole-brain radiation therapy failure: who actually dies of their brain metastases? Int J Radat Oncol Biol Phys 92 : 1008-1015, 2015

8. Mariya Y, Sekizawa G, Matsuoka Y, Seki H, Sugawara T, Sasaki Y : Repeat stereotactic radiosurgery in the management of brain metastases from non-small cell lung cancer. Tohoku J Exp Med 223 : 125-131, 2011

9. Marvaso G, Barone A, Vaccaro C, Bruzzaniti V, Grespi S, Scotti V, et al. : Repeat stereotactic radiosurgery in the management of brain metastases from NSCLC: a case report and review of the literature. Oncol Lett $6: 897-900,2013$

10. McKay WH, McTyre ER, Okoukoni C, Alphonse-Sullivan NK, Ruiz J, Munley MT, et al. : Repeat stereotactic radiosurgery as salvage therapy for locally recurrent brain metastases previously treated with radiosurgery. J Neurosurg 127 : 148-156, 2017

11. Shuto T, Fujino $H$, Inomori $S$, Nagano $H$ : Repeated gamma knife radiosurgery for multiple metastatic brain tumours. Acta Neurochirurg (Wien) 146 : 989-993; discussion 993, 2004

12. Sperduto PW, Wang M, Robins HI, Schell MC, Werner-Wasik M, Komaki R, et al. : A phase 3 trial of whole brain radiation therapy and stereotactic radiosurgery alone versus WBRT and SRS with temozolomide or erlotinib for non-small cell lung cancer and 1 to 3 brain metastases: Radiation Therapy Oncology Group 0320. Int J Radat Oncol Biol Phys 85 : 13121318, 2013

13. Yamamoto M, Serizawa T, Shuto T, Akabane A, Higuchi Y, Kawagishi J, et al. : Stereotactic radiosurgery for patients with multiple brain metastases (JLGK0901): a multi-institutional prospective observational study. Lancet Oncol 15 : 387-395, 2014

14. Yamanaka K, Iwai Y, Yasui T, Nakajima H, Komiyama M, Nishikawa M, et al. : Gamma knife radiosurgery for metastatic brain tumor: the usefulness of repeated gamma knife radiosurgery for recurrent cases. Stereotact Funct Neurosurg 72 Suppl $1: 73-80,1999$ 